\title{
Topography of the merit function landscape in optical system design
}

\author{
Eco van Driel, Florian Bociort, Alexander Serebriakov* \\ Optics Research Group, Delft University of Technology \\ Lorentzweg 1, NL - 2628 CJ Delft, The Netherlands
}

\begin{abstract}
We have shown recently that, when certain quite general conditions are satisfied, the set of local minima in the optical merit function space forms a network where they are all connected through optimization paths generated from saddle points having a Morse index of 1. A new global optimization method, that makes use of this linking network to systematically detect all minima, is presented. The central component of this new method, the algorithm for saddle point detection, is described in detail and we show that the initialization of this algorithm has a significant impact on the performance. For a simple global optimization search (Cooke triplet) several representation forms of the network of the corresponding set of local minima are presented. These representations, which can be visualized in two dimensions, are independent of the dimensionality of the design space so that they can provide insight into the topography of merit function landscapes of arbitrary dimensionality.
\end{abstract}

Keywords: global optimization, saddle point, Morse index, network, optical system design

\section{INTRODUCTION}

Present day optical design software makes use of powerful global optimization algorithms ${ }^{1,2,3,4}$. Unfortunately, the minima detected with such algorithms are given only as isolated points in the parameter space without any information about the topography of the merit function space between them. Recently, we have shown that optimization paths generated from a special type of saddle points (saddle points having a Morse index of 1) connect all minima in the optical merit function space .

In this paper, we give additional details about the method used to detect the saddle points. We also introduce several representation forms for the topography of the network structure of merit function spaces of arbitrary dimensions. These representation forms can be used to visualize the relationship between the minima while ignoring issues such as the dimensionality or local characteristics of the merit function space. These networks can become an important tool for the study of complex design problems encountered in optics.

In section 2 it is shown that the relationship between the various minima can be derived only by considering the equimagnitude surfaces around minima and saddle points having a Morse index of one. In Section 3 we describe a new method for global optimization based on the detection of the network of minima. This method makes use of the network structure to systematically find all minima and saddle points having a Morse index of 1 . The detection of saddle points which forms the central component of our method is discussed in section 4 . We will show that saddle points having a Morse index of 1 can be detected by using only the local optimization engine of optical design software. We also show that the initialization of such an algorithm has a significant impact on the efficiency of the algorithm. In section 5, several representation forms of the networks of minima are introduced. These representations can be used as tools for the analysis of the topography of merit function spaces of arbitrary dimensions.

*a.serebriakov@tnw.tudelft.nl; phone+31 15 2784250; fax +31 152788105 


\section{CONNECTED NETWORKS OF MINIMA}

We consider an optimization problem with continuous variables and assume a continuous merit function $\mathrm{f}$ that is bounded from below. (In optics, a merit function that is defined as a sum of squares satisfies this requirement.) A point in the solution space is described by a vector $\mathbf{x}=\left(\mathrm{x}_{1}, \mathrm{x}_{2}, \ldots, \mathrm{x}_{\mathrm{N}}\right)$ whose components are the $\mathrm{N}$ optimization variables. For our current discussion, the equimagnitude surfaces in a small neighborhood around the critical points of $\mathrm{f}$ are of particular interest. At a critical point, the gradient of $\mathrm{f}$ vanishes and the equimagnitude surfaces may be written as

$$
\sum A_{i j} \hat{x}_{i} \hat{x}_{j}=\text { const }
$$

where the circumflex denotes the values of the optimization variables in a translated coordinate system that has its origin at the critical point, and where $\boldsymbol{A}$ is the matrix of second-order derivatives computed at the critical point

$$
A_{i j}=\frac{\partial^{2} f}{\partial \hat{x}_{i} \partial \hat{x}_{j}}
$$

As known from linear algebra, the coordinate system can be rotated in such a way that the quadratic form on the lefthand side of Eq. (1) contains only squares of the variables in the new coordinate system $\bar{x}$. The equimagnitude surfaces around the critical point are then given by

$$
\sum \lambda_{i} \bar{x}_{i}^{2}=\text { const }
$$

where the new coordinates are measured along the eigenvectors of the matrix $\boldsymbol{A}$ and the factors $\lambda$ are the eigenvalues corresponding to these eigenvectors. As $\boldsymbol{A}$ is a square symmetric matrix, the eigenvalues are real and the eigenvectors corresponding to different eigenvalues are mutually orthogonal.

Assuming that all eigenvalues are non-zero, the Morse index $(M I)$ of a critical point is defined as the number of negative eigenvalues in Eq. (3). A negative eigenvalue means that along the direction of the corresponding eigenvector the critical point is a maximum. Thus local minima have $M I=0$ (all eigenvalues are positive so that there do not exist ascending directions at such a point) whereas local maxima have $M I=N$. Critical points having a Morse index between 1 and N-1 are referred to as saddle points. Equimagnitude surfaces around a saddle point with $M I=1$ consist of one surface for merit function values higher than that of the saddle point while they consist of two separated surfaces for equimagnitude values lower than that of the saddle point (see Fig. 1).
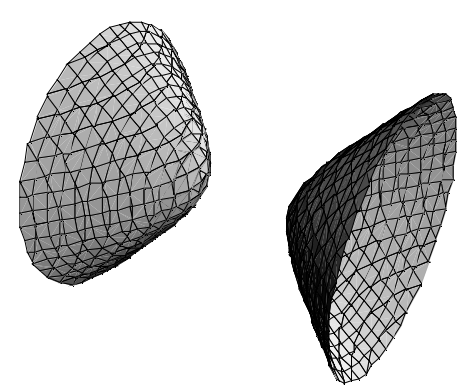

a)

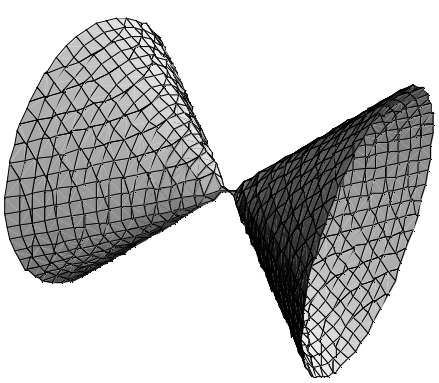

b)

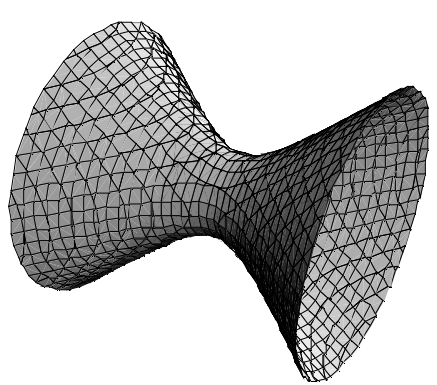

c)

Figure 1 Typical behavior around a saddle point having a Morse index of one. The merit function value at the saddle point is $f_{s p}$. a) for $f<f_{s p}$, we have two surfaces. b) for $f=f_{s p}$ the two surfaces only touch at the saddle point and encircle the surfaces drawn in a). c) for $f>f_{s p}$ one surface encircles the saddle point and all other surfaces with smaller equimagnitude values. 
Consider now the merit function landscape shown in Fig 2. First note that the surface with merit function value $f=f_{a}$ encircles the complete set of minima. However, Eq. (3) predicts that the equimagnitude surfaces around each minimum are ellipsoids that eventually reduce to a single point at the minimum they encircle. For a continuous merit function space, the encircling surface will thus be split in a series of equimagnitude surfaces that each ultimately encircle only one minimum. We will show that the splitting of one encircling surface into two separated surfaces occurs at saddle points having a Morse index of 1 .

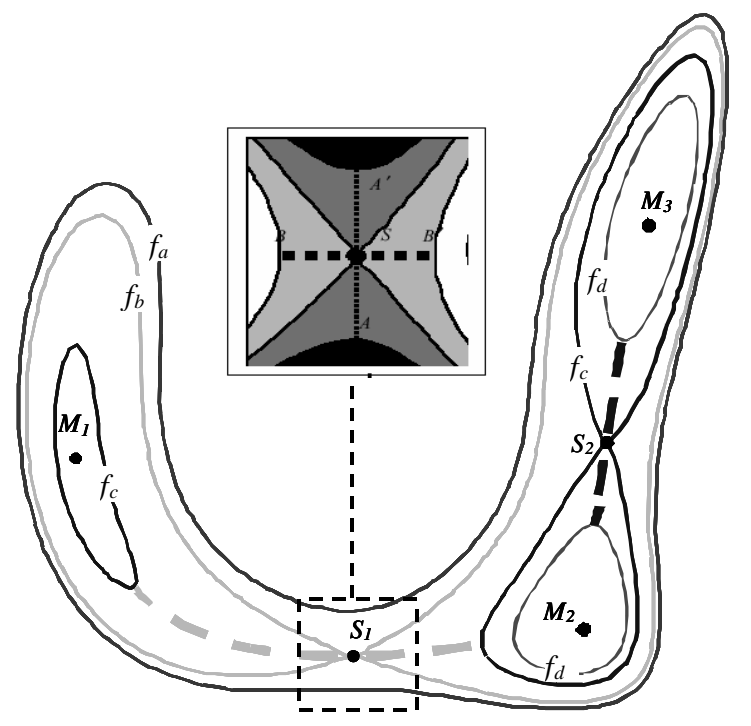

Figure 2 Three local minima $\left(\mathrm{M}_{1}, \mathrm{M}_{2}\right.$ and $\left.\mathrm{M}_{3}\right)$ can be distinguished that are each encircled by a number of equimagnitude surfaces. The merit function values at the three minima are lower than those of the encircling equimagnitude surfaces with $f_{d}<f_{c}<f_{b}<f_{a}$. The two surfaces with merit function value $f_{c}$ can be connected through optimization paths (dashed lines) generated from the point $\mathrm{S}_{1}$. Similarly, the $f_{d}$ surfaces are connected through paths from $\mathrm{S}_{2}$. In the enlarged detail around the point $S_{1}$, light gray indicates lower and dark gray indicates higher values of the merit function.

In Fig. 2, the equimagnitude surface with $f=f_{b}$ is the smallest surface that still encircles the complete set of minima. For lower values, e.g. $f=f_{c}$, we have two separated equimagnitude surfaces that each encircle a sub-set of the total number of minima. If we now consider a surface with a merit function value $f=f_{b}$, slightly lower than $f_{b}$, the corresponding surface is split. Let BB' be the two points on the separated parts of the equimagnitude surface for which the length of the segment BB' is minimal. Obviously along BB', the point $\mathrm{S}_{1}$ is a maximum. A surface with a value $f_{a}$, slightly higher than $f_{b}$ encircles the surface with value $f_{b}$. For any line perpendicular to BB' and passing through $S_{1}$ we then have a minimum at $S_{1}$. Since this is valid for any choice of AA' in the hyperplane orthogonal to BB', $S_{1}$ is a saddle point having a Morse index of 1 . At $S_{1}$, we can now perform two local optimizations. A local optimization initiated on the left side of $S_{1}$ then converges to $M_{1}$ while a local optimization initiated on the right side of $S_{1}$ converges to one of the minima encircled by the equimagnitude surface containing $\mathrm{M}_{2}$ and $\mathbf{M}_{3}$. (For the merit function landscape in Fig. 2, the optimization will most likely converge towards $\mathrm{M}_{2}$.) Hence, the optimization paths from saddle point $\mathrm{S}_{1}$ connect two distinct minima. For similar reasons, the point $S_{2}$ is also a saddle point having a Morse index of 1 and this saddle point connects $\mathrm{M}_{2}$ with $\mathrm{M}_{3}$. The set of minima inside an encircling surface are thus connected through optimization paths generated from the saddle points having a Morse index of 1 .

It is important to note that this linking network is independent of the exact shape of the equimagnitude surfaces and the dimensionality of the merit function space. From a topological point of view, two surfaces are considered equivalent if there exists a continuous deformation that transforms one surface into the other one. Therefore, the network of minima represents changes in the topology of the merit function landscape. However, we only consider topological changes for which the equimagnitude surfaces are split for decreasing merit function values (saddle points with $M I=1$ ) and topological changes for which equimagnitude surfaces vanish (local minima). Other topological changes, which might be of interest for other purposes, occur at critical points having a Morse index higher than 1. 


\section{GLOBAL OPTIMIZATION}

As shown in the previous section, the local minima within an encircling equimagnitude surface ${ }^{6}$ are all connected through optimization paths generated from the saddle points that have a Morse index of 1 . Starting form such a saddle point, two distinct local minima can be obtained by means of local optimization only. By detecting the saddle points that connect a given minimum with the remainder of the network, we can systematically detect the complete network of minima. Once the complete network has been detected, not only the global minimum is known but also the relation between the various minima. A basic algorithm for the detection of the complete network of minima is presented in Fig. 3. Algorithms for finding new local minima based on saddle point detection that have some similarities with our method can be found in papers by Barkema and Mousseau ${ }^{7-9}$ and by D. Wales ${ }^{10-11}$ where the energy landscape of many atoms is studied.

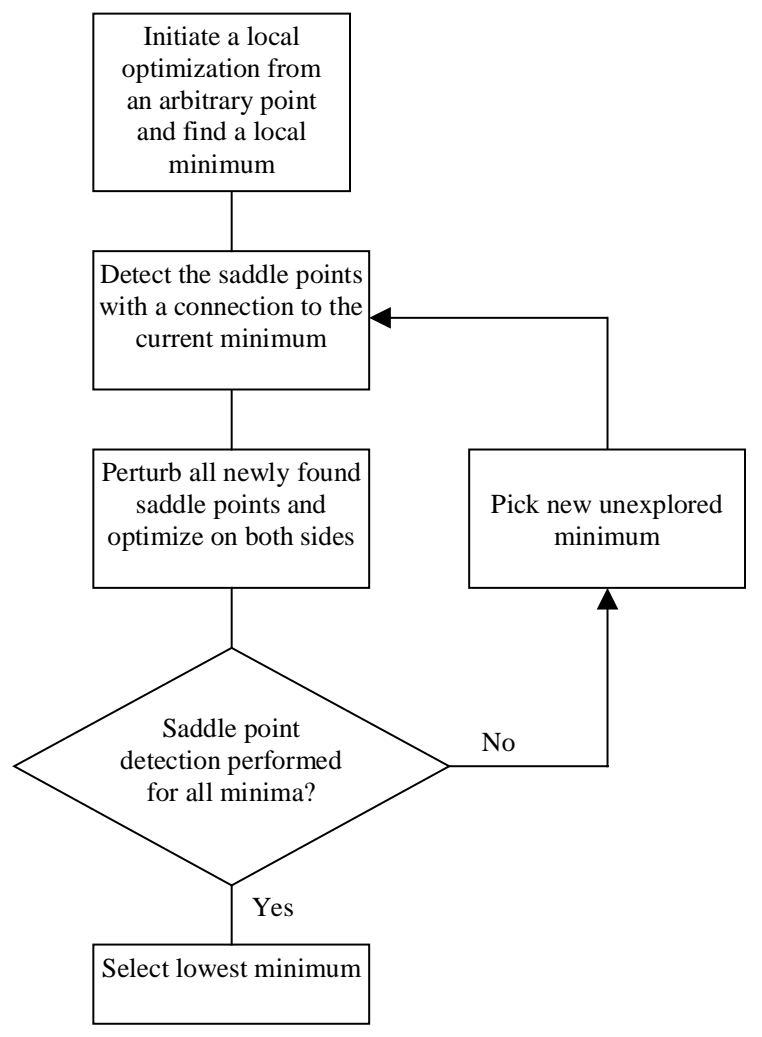

Figure 3 Flow chart for saddle point based global optimization.

The procedure for global optimization presented in Fig. 3 is essentially different from other global optimization strategies as the search for the global minimum is now split in two distinct stages: a local minimization from saddle points and a local saddle point search from minima (i.e. a search for the saddle points connected to the given minimum). Ideally, repeated use of these two stages would find all Morse index one saddle points and all minima. We thus try to find all minima in a structured manner where we only consider direct connections between minima and saddle points. For a given minimum, we only need to detect those saddle points for which the optimization paths lead towards that minimum. Other saddle points can then be located from the other minima. In Fig. 2 it is thus not required to find $S_{2}$ while using $M_{1}$ as initial point for the saddle point detection. It is sufficient to find $S_{1}$ so that the local optimization from $S_{1}$ results into the location of $M_{2}$. However, while initiating a search from $M_{2}$, it is required to find both $S_{1}$ and $S_{2}$. In addition to finding the complete set of minima, such a search also provides additional insight into the topography of the merit function space as will be shown in section 5 . 


\section{SADDLE POINT DETECTION}

Although we may safely ignore saddle points having a Morse index higher than one, detecting all saddle points that connect a given minimum to the rest of the network remains a challenging task. Due to the high dimensionality of the optical merit function space and the non-analytical form of the merit function, methods that require the frequent computation and inversion of the Hessian matrix are not well suited, as they are too intensive computationally. Furthermore, the frequent usage of constraints in optics requires an algorithm for saddle point detection that is capable of efficiently dealing with (in-) equality constraints.

We have developed an algorithm for saddle point detection that is based on constrained local optimization. Consider first an initial point $\mathbf{x}_{\mathbf{0}}$ which can be a minimum or a Morse index one saddle point. A new coordinate system $\hat{\mathbf{x}}$ is now defined with this initial point in the origin. We also choose a direction $\mathbf{s}$ and consider the set of hyperplanes orthogonal to $\mathbf{S}$

$$
s_{1} \hat{x}_{1}+s_{2} \hat{x}_{2}+\ldots+s_{N} \hat{x}_{N}=t
$$

where $\mathrm{t}$ gives the distance between the hyperplane and the initial point $\mathbf{x}_{\mathbf{0}}$ measured along the normal that passes through $\mathbf{x}_{\mathbf{0}}$. For a given value of $t$ we can then compute the constrained minimum of $f$ in the set of hyperplanes given by Eq.4. For an appropriate choice of $\mathbf{s}$, these constrained minima form a continuous path connecting the saddle point with the minimum as shown in Fig. 4. Let $\mathbf{x}_{\mathrm{s}}(\mathrm{t})$ and $\mathrm{F}_{\mathrm{s}}(\mathrm{t})$ be the position vector and the value of $\mathrm{f}$ corresponding to a constrained minimum in a hyperplane defined by Eq.(4). Then the points on a given trajectory are uniquely defined by the requirement that the gradient of $f$ is parallel to $\mathbf{s}$ for all $\mathbf{x}_{\mathbf{s}}(\mathrm{t})$.

Consider now a trajectory $\mathbf{x}_{\mathbf{s}}(\mathrm{t})$ generated with a minimum as initial point. For any choice of $\mathbf{s}$ we have a minimum at $F_{s}(0)$ while a gradual increase of $t$ then results into an increase of $F_{s}(t)$. A neighboring saddle point is detected when $\mathrm{F}_{\mathrm{s}}(\mathrm{t})$ reaches a maximum and $\mathbf{x}_{\mathrm{s}}(\mathrm{t})$ is continuous for $0<\mathrm{t}<\mathrm{t}_{\max }$ where $\mathrm{t}_{\max }$ is the distance corresponding to the maximum of $\mathrm{F}_{\mathrm{s}}(\mathrm{t})$. This procedure is illustrated in Fig. 5 where the hyperplanes are shifted into an arbitrary direction.
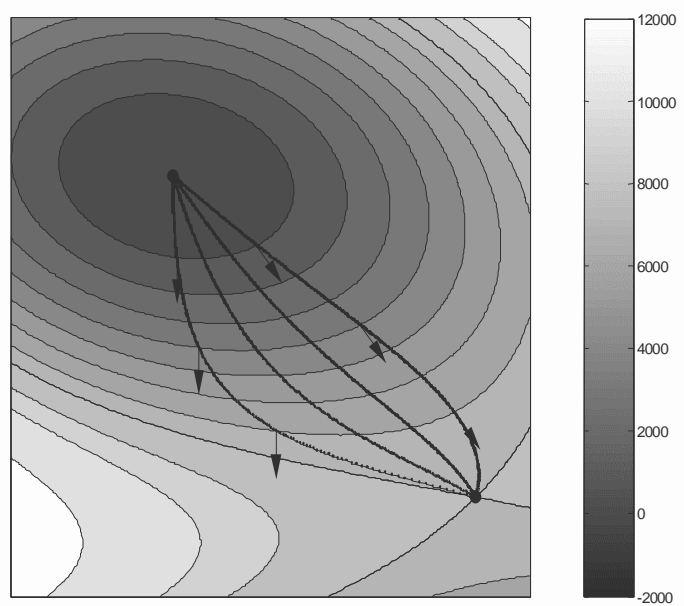

Figure 4 Paths between a local minimum and a saddle point generated by means of a constrained local minimization orthogonal to a direction $\mathbf{s}$. Each of these paths is defined with a different search direction. Due to the local minimization orthogonal to $\mathbf{s}$, the gradient direction is parallel to $\mathbf{s}$ for all points on a given path. The arrows indicate the direction of the gradient for some points on the paths.

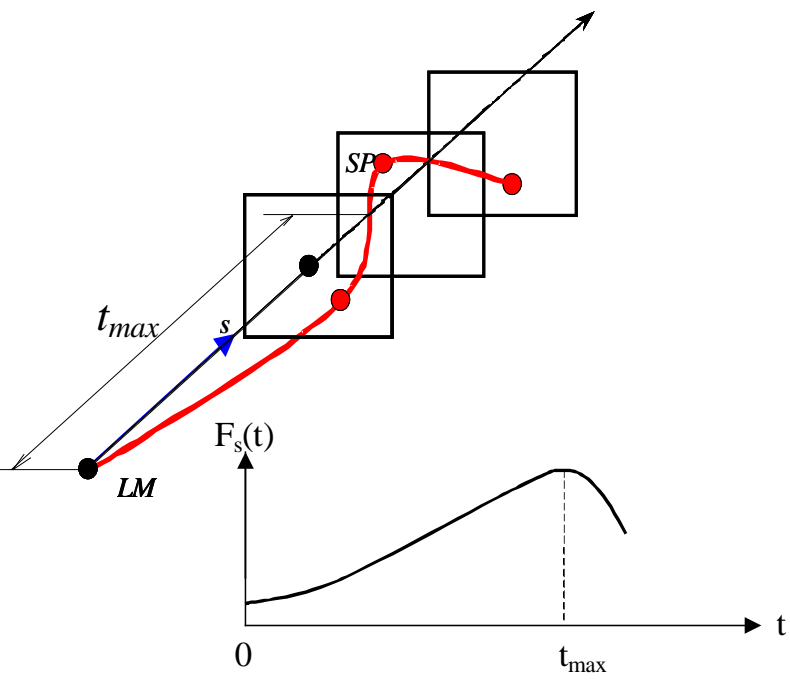

Figure 5 Saddle point detection by using constrained local minimization. Starting at a local minimum $F_{s}(t)$ increases until it reaches a maximum at a saddle point. Although $\mathbf{x}_{\mathbf{s}}(\mathrm{t})$ may be a complicated trajectory through the design space, the saddle point detection is now effectively a one dimensional maximization problem for $\mathrm{F}_{\mathrm{s}}(\mathrm{t})$. 
At a minimum, any direction $\mathbf{s}$ leads initially to an increase of $F_{s}$. However, a saddle point can only be reached if $\mathbf{s}$ is such that the saddle point is a maximum into the direction of $\mathbf{s}$ while it is a minimum orthogonal to that direction. For a saddle point with a given Hessian matrix $\boldsymbol{A}$ the search is successful and $\mathbf{x}_{\mathbf{s}}(\mathrm{t})$ passes through the saddle point only if

$$
\sum A_{i j} s_{i} s_{j}<0
$$

If this condition is not met, the path becomes discontinuous and does not lead to a neighboring saddle point. However, new local minima (situated further away in the network) can still be discovered in this way.

One of the most important aspects for successful saddle point detection is a proper initialization of the algorithm. The number of searches performed at each minimum has to be determined as well as the search directions. We will show that choosing search directions randomly is not an optimal solution because the resulting trajectories will be initially concentrated in a narrow cone around the eigenvector with smallest eigenvalue. We first discuss the appropriate choice of search directions and after that, the required number of searches will be considered.

Without prior knowledge of the location of the connecting saddle points, any direction of search may be defined for starting the search at a minimum. However, by initiating multiple searches from a given minimum, we want to explore different regions of the merit function space. The probability of detecting all connecting saddle points can then be maximized by generating paths with a maximum spatial separation. To study the spatial separation between paths generated with different directions of search, we consider the quadratic region around a minimum where the equimagnitude surfaces may be approximated by ellipsoids. In this region, the behavior of the merit function is completely determined by the Hessian matrix at the minimum. For a given Hessian, $\boldsymbol{A}$, the gradient is then given by

$$
\nabla f\left(\mathbf{x}_{\mathbf{M}}+\mathbf{\Delta} \mathbf{x}\right)=A \mathbf{\Delta} \mathbf{x}=\Lambda \overline{\mathbf{x}}
$$

where the diagonal matrix $\Lambda$ contains the eigenvalues of the Hessian matrix and the components of the vector $\bar{x}$ are measured along the directions of the eigenvectors of the Hessian matrix.

The minimization orthogonal to $\mathbf{s}$ ensures that all points on a path generated with a given direction of search have a gradient direction given by $\mathbf{s}$. Hence, these points are given by

$$
\overline{\mathbf{x}}_{\mathbf{s}}(t)=\gamma(t) \Lambda^{-1} \mathbf{s}
$$

where $\gamma$ is a scaling factor depending on the distance, $t$ as defined in Eq.(4). In the eigenvector basis, the components of the path vector are then given by

$$
\bar{x}_{s, i}(t)=\gamma(t) \lambda_{i}^{-1} s_{i} \quad, i=1 \ldots N
$$

Note that when some eigenvalue is very small, the component of the path vector along the corresponding eigenvector tends to be much larger than the other components.

In the two-dimensional example shown in Fig. 6, a search direction $\mathbf{s}$ is defined with an angle $\alpha$ with respect to the first eigenvector, i.e. the eigenvector with smallest eigenvalue. The corresponding search path $\mathbf{x}_{\mathbf{s}}(\mathrm{t})$ is now given by Eq.(7) and this path makes an angle $\theta$ with respect to the first eigenvector.

With these definitions we have

$$
\tan \theta=\frac{\overline{\mathbf{x}}_{2}}{\overline{\mathbf{x}}_{1}}, \tan \alpha=\frac{\mathbf{s}_{2}}{\mathbf{s}_{1}}
$$




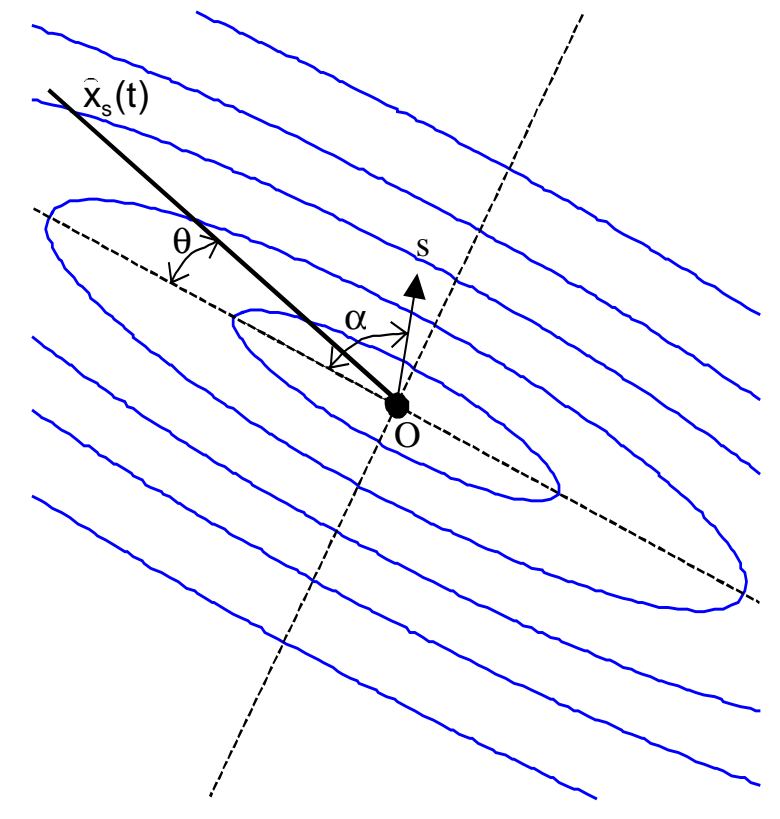

Figure 6 Path $\mathbf{x}_{\mathbf{s}}(\mathrm{t})$ generated with an arbitrary direction $\mathbf{s}$. The angles $\alpha$ and $\theta$ are defined with respect to the eigenvector with smallest eigenvalue (the longest axis of the ellipse).

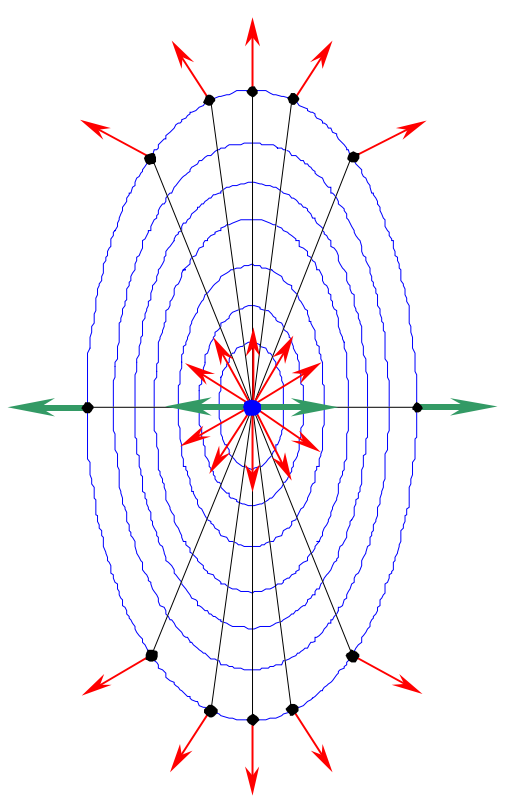

Figure 7 Paths generated for a set of directions $\mathbf{s}$ defined with an angular separation of 30 degrees between them. The arrows indicate the direction of search. Note that most paths are concentrated around the eigenvector with smallest eigenvalue.

It follows then from Eq.(8) that the two angles are related through

$$
\tan \theta=\frac{\lambda_{1}}{\lambda_{2}} \tan \alpha
$$

where $\lambda_{1}$ and $\lambda_{2}$ are the eigenvalues of the Hessian matrix at the local minimum. It follows from Eq. (10) that for very elongated ellipsoids (i.e. $\lambda_{1}<<\lambda_{2}$ ) most paths tend to concentrate in a narrow cone around the eigenvector with smallest eigenvalue (see Fig. 7). However, we can use the direction of the second eigenvector of the Hessian matrix (thick horizontal arrow) to escape from the narrow cone around the first eigenvector in order to explore different regions of the merit function space. For each eigenvector two searches in opposite directions can be performed so that we have a total number of four independent searches in two dimensions.

In general it follows from $\mathrm{Eq}(8)$ that by defining the search directions such that they correspond with the directions of different eigenvectors of the Hessian matrix the paths are independent of the eigenvalues of the Hessian matrix. The paths then initially follow the directions of the eigenvectors and they are mutually orthogonal. In the case of an Ndimensional design space, we can thus define $\mathrm{N}$ directions of search corresponding with the directions of the eigenvectors. For each of the eigenvectors, the search can be performed in opposite directions so that the total number of searches is given by $2 \mathrm{~N}$. The directions of the eigenvectors at a minimum can be determined by means of local optimization $^{5}$.

Although a number of only $2 \mathrm{~N}$ searches might seem insufficient when compared to the number of saddle points that can be encountered in a typical design problem, it should be realized that these $2 \mathrm{~N}$ searches are performed at each minimum. As noted earlier, at each of these minima, only those saddle points having a direct linkage with the minimum need to be detected. According to our current experience, most saddle points have been detected several times indicating that $2 \mathrm{~N}$ searches are sufficient. If more than $2 \mathrm{~N}$ searches are desired, Eq. (8) can also be used to maximize 
the separation between these paths by taking into account the eigenvalues at a minimum. However, for more complex optimization problems, it might be more efficient to use less than $2 \mathrm{~N}$ search directions. As the main difference between the Hessian matrix at a minimum and at a saddle point with a Morse index of 1 is the sign of one of the eigenvalues, it might be sufficient to use only those eigenvectors for which the eigenvalues are relatively low as we can expect that those eigenvectors have a higher probability of becoming negative.

\section{RESULTS}

We have implemented the algorithm described in the preceding sections in the macro language of the commercial optical design program Code V. Here, we present several results obtained for merit function spaces of triplets. In our first example the triplet forms an image of an object at infinity and we have used the first five curvatures as variables. The image plane was placed at its paraxial position and the curvature of the last surface has been solved to keep a constant effective focal length. The default Code $\mathrm{V}$ merit function (which is based on transverse ray aberrations) was used. The resulting network, which is presented in Fig. 8, consists of 18 local minima and 20 saddle points. In this network, each node represents a point in the five-dimensional design space and the lines connecting nodes represent optimization paths that have been generated from the saddle points. As expected, the local minima form a network where they are all linked through optimization paths generated from the saddle points. This network, which has been detected in five dimensions, can be visualized in a two-dimensional graph. This allows us to examine the relationship between the various minima independently of the dimensionality of the merit function space.

The network represented in Fig. 8 has an excess of saddle points as only 17 saddle points are required to form a network where all 18 minima are connected. For example, the saddle point having a merit function value of 54390 can be removed without disconnecting the network ${ }^{11}$. By removing all such superfluous saddle points, we obtain the "essential" topography of the merit function space as presented in Fig. 9. The acyclic network thus formed shows the minimum barriers in merit function value that needs to be overcome in order to move from one minimum to another one. From Fig. 9 we can also derive a binary-tree representation where the splitting of the equimagnitude surfaces is represented (Fig. 10). This binary tree can be used to visualize how the equimagnitude surfaces split as a function of decreasing merit function values. Such a representation is less sensitive to the type of local optimization algorithm used to generate the paths from the saddle points towards the minima.

Even in the case of a constrained merit function space, we can apply our algorithm to detect the network of minima. For instance, we have added an additional constraint such that the curvature of the fifth surface is larger than zero. The corresponding network of minima is presented in Fig. 11 and this network shows a remarkable similarity with the network presented in Fig. 9. Those parts of the network for which the constraints have not been violated are identical. However, saddle points and minima are now also found on the constraint while other parts of the unconstrained network do not exist anymore.

Our current experience has shown that the searches into the direction of the eigenvector with smallest eigenvalue find slightly less than $50 \%$ of the saddle points. To find all saddle points, the eigenvectors with higher eigenvalues are also important. In the searches for the unconstrained Cooke triplet, the different eigenvalues of the Hessian matrix at a minimum can differ significantly. For one of the minima, the highest eigenvalue was approximately 25,000 times higher than the lowest eigenvalue of the Hessian matrix. Mostly, the ratio between the highest and lowest eigenvalue at a minimum ranges between 500 and 6000 so that the initialization of the algorithm for saddle point detection is extremely important in optics. 


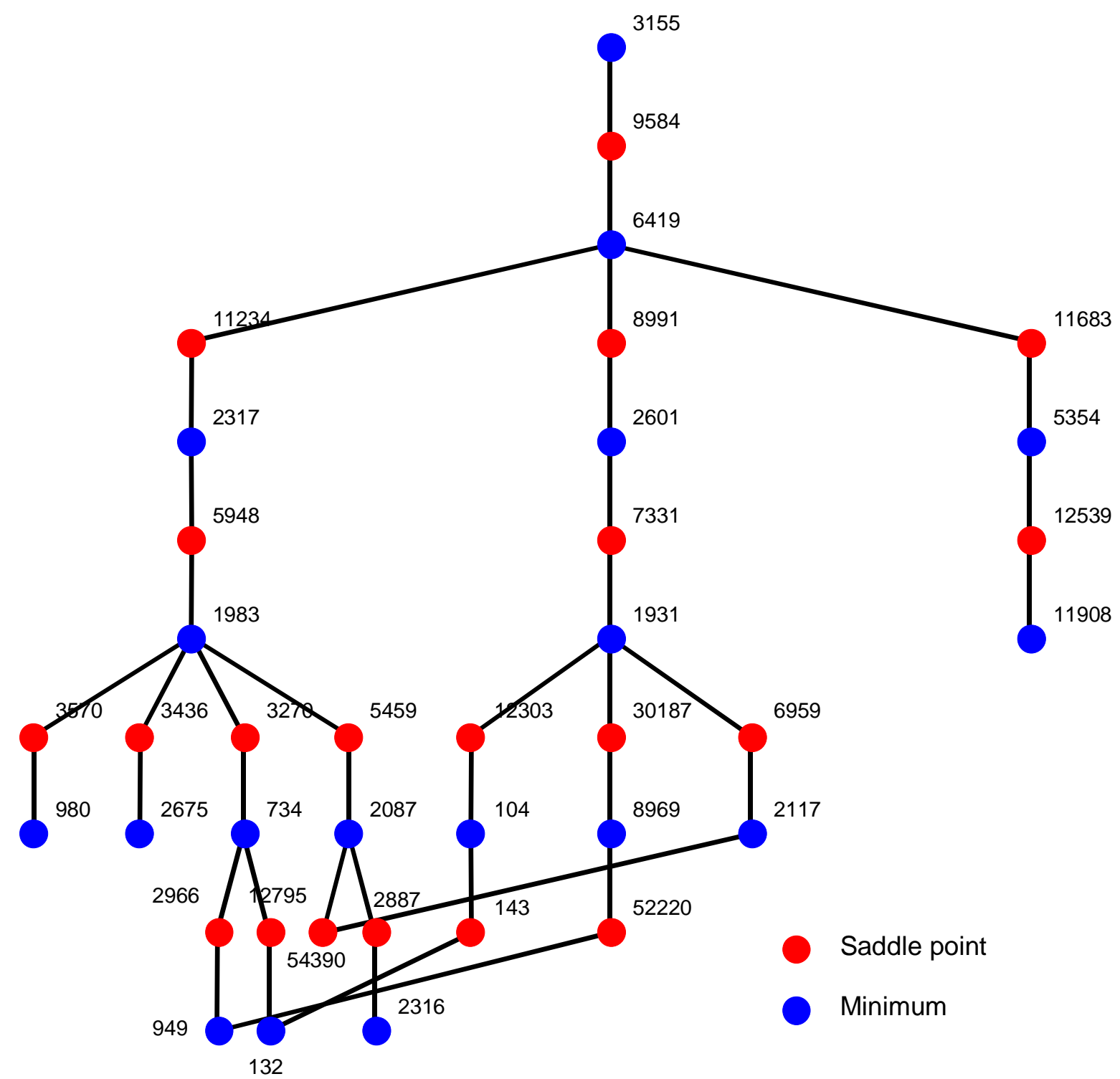

Figure 8 Network of the global search corresponding to the Cooke triplet. The nodes represent optical systems corresponding to the saddle points and local minima. Nodes are connected through optimization paths generated from the saddle points. 


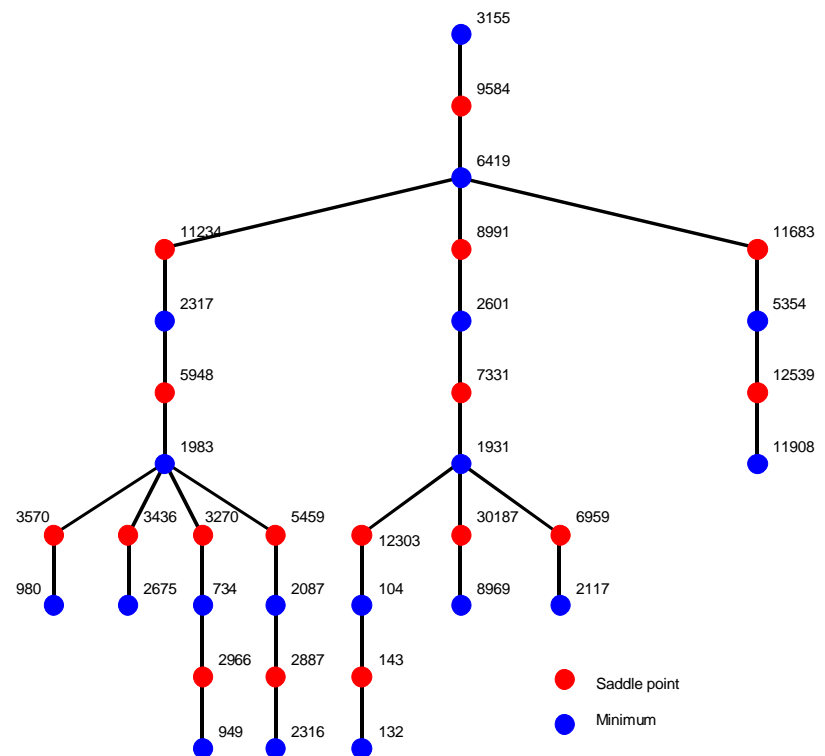

Figure 9 Essential network of the global search corresponding to the Cooke triplet. The minimum barrier in merit function value that needs to be overcome in order to move from one minimum to another one is determined by the saddle point with highest merit function value that separates these minima.

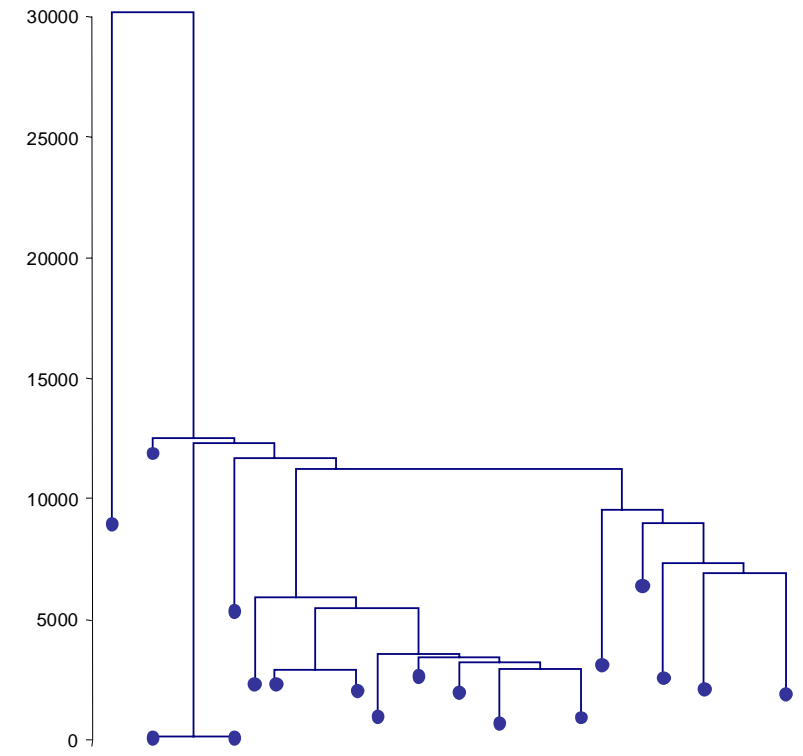

Figure 10Tree structure of the global search corresponding to the Cooke triplet. The splitting of the equimagnitude surfaces is represented as a function of the merit function value. The branching points are the saddle points and the endpoints are the local minima

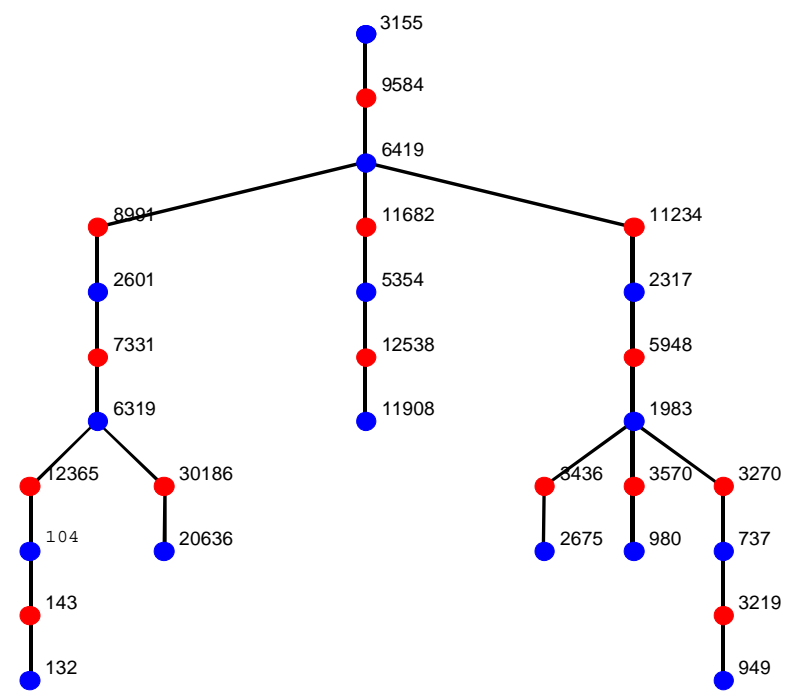

Figure 11Essential network of the global search corresponding to the constrained Cooke triplet. Large parts of the network are identical to the unconstrained network. (Note however, that the branches are drawn in a different order.) 


\section{CONCLUSIONS}

We have shown that the local minima in the optical merit function space form a network where they are all linked through optimization paths generated from saddle points having a Morse index of 1 . This feature has encouraged us to develop a new type of global optimization that can be applied to systematically approach the problem of detecting multiple minima. We have also shown that the Morse index 1 saddle points can be detected by a constrained local optimization. Arbitrary search paths generated with our algorithm for saddle point detection tend to concentrate in a narrow cone around the eigenvector with smallest eigenvalue while search paths generated into the directions of the eigenvectors are mutually orthogonal so that a maximum spatial separation between the search paths can be obtained. Due to the use of local optimization for the saddle point detection, we have been able to include constraints in the optimization problem.

For a relatively simple global optimization problem, the network of minima has been detected and two presentation forms of this network where introduced. These representations allow a comprehensible view of the relationship between the various minima without having to deal with aspects such as the dimensionality of the optimization problem. These networks could thus be of use for the analyses of complex optical design problems.

\section{ACKNOWLEDGEMENTS}

We would like to thank Joseph Braat and Jos Thijssen for stimulating discussions and suggestions. We also acknowledge the use of an educational license of CODE V.

\section{REFERENCES}

1. G. W. Forbes and A. E. W. Jones, "Towards global optimization with adaptive simulated annealing", Proc. SPIE 1354, 144-151, 1991

2. T. G. Kuper and T. I. Harris, "Global optimization for lens design - an emerging technology", Proc. SPIE 1780,1428, 1992

3. K. E. Moore, "Algorithm for global optimization of optical systems based on genetic competition", Proc. SPIE 3780, 40-47, 1999

4. M. Isshiki, H. Ono, K. Hiraga, J. Ishikawa, S. Nakadate, "Lens design: Global optimization with Escape Function", Optical Review (Japan), 6, 463-470, 1995

5. F. Bociort, E. van Driel, A. Serebriakov, "Network structure of the set of local minima in optical system optimization", to be published in Proc. SPIE 5174, 2003

6. It seems that, if the encircling condition is not satisfied automatically, in many optimization problems with continuous variables this condition can be enforced by modifying the problem adequately (e.g. by using inequality constraints). Fictitious local minima and saddle points might appear in this way. However, the real minima together with the fictitious ones will then form a connected network.

7. G. T. Barkema, N. Mousseau, "Event-Based Relaxation of Continuous Disordered Systems", Phys. Rev. Lett. 77, 4358, 1996

8. N. Mousseau, G.T. Barkema, "Travelling through potential energy landscapes of disordered materials: The activation-relaxation technique", Phys. Rev. E 57, 2419, 1998

9. G. Wei, N. Mousseau, P. Derreumaux, "Exploring the energy landscape of proteins: A characterization of the activation relaxation technique", J. Chem. Phys. 117, 11379, 2002

10. D. J. Wales, "Rearrangements of 55 atom Lennard-Jones and $\left(\mathrm{C}_{60}\right)_{55}$ Clusters", J. Chem. Phys. 101, 3750,1994

11. J. P. K. Doyle, D.J. Wales, "Surveying a potential energy surface by eigenvector-following", Z. Phys. D 40, 194, 1997

12. E. van Driel, Network structure of local minima in optical system design, Master of Science thesis, TU Delft, Optics Research Group, 2003 